\title{
Role of Chondroitin Sulfate Proteoglycans in Axonal Conduction in Mammalian Spinal Cord
}

\author{
Arsen S. Hunanyan, ${ }^{1,2}$ Guillermo García-Alías, ${ }^{3}$ Valentina Alessi, ${ }^{1,2}$ Joel M. Levine, ${ }^{2}$ James W. Fawcett, ${ }^{3}$ \\ Lorne M. Mendell, ${ }^{2}$ and Victor L. Arvanian ${ }^{1,2}$ \\ ${ }^{1}$ Northport Veterans Affairs Medical Center, Northport, New York 11768, ${ }^{2}$ Department of Neurobiology and Behavior, State University of New York at \\ Stony Brook, Stony Brook, New York 11794-5230, and ${ }^{3}$ University of Cambridge Centre for Brain Repair, Department of Clinical Neurosciences, Cambridge \\ CB2 0PY, United Kingdom
}

Chronic unilateral hemisection (HX) of the adult rat spinal cord diminishes conduction through intact fibers in the ventrolateral funiculus (VLF) contralateral to HX. This is associated with a partial loss of myelination from fibers in the VLF (Arvanian et al., 2009). Here, we again measured conduction through the VLF using electrical stimulation while recording the resulting volley and synaptic potentials in target motoneurons. We found that intraspinal injection of chondroitinase- $\mathrm{ABC}$, known to digest chondroitin sulfate proteoglycans (CSPGs), prevented the decline of axonal conduction through intact VLF fibers across from chronic T10 HX. Chondroitinase treatment was also associated with behavior suggestive of an improvement of locomotor function after chronic HX. To further study the role of CSPGs in axonal conduction, we injected three purified CSPGs, NG2 and neurocan, which increase in the vicinity of a spinal injury, and aggrecan, which decreases, into the lateral column of the uninjured cord at T10 in separate experiments. Intraspinal injection of NG2 acutely depressed axonal conduction through the injected region in a dose-dependent manner. Similar injections of saline, aggrecan, or neurocan had no significant effect. Immunofluorescence staining experiments revealed the presence of endogenous and exogenous NG2 at some nodes of Ranvier. These results identify a novel acute action of CSPGs on axonal conduction in the spinal cord and suggest that antagonism of proteoglycans reverses or prevents the decline of axonal conduction, in addition to stimulating axonal growth.

\section{Introduction}

Many patients with partial spinal cord injuries and preserved axons may nevertheless have no descending motor or ascending sensory function from segments below the injury. Various factors, including inflammation and demyelination, have been proposed to explain the lack of conduction in surviving axons (Blight, 1983). Recently, we found that a lateral hemisection lesion (HX) of the adult rat spinal cord induces failure of axonal conduction through large intact axons contralateral to the HX, beginning 1 week after injury and persisting for at least 14 weeks (Arvanian et al., 2009). The initiation of these physiological deficits coincided with the time that the elevated level of chondroitin sulfate proteoglycans (CSPGs) in tissue surrounding the HX reached its peak (Lou et al., 2008). A similar time schedule for CSPG accumulation around the injury site has been reported in other spinal cord injury (SCI) models (Lemons et al., 1999; Leonard et al., 2003; Davies et al., 2004; Iaci et al., 2007; García-Alías et al., 2008).

Behavioral and anatomical studies suggest that the elevated level of CSPGs in and around the glial scar is a major obstacle for recovery after SCI (Snow et al., 1990; Jones et al., 2002; Galtrey

Received Sept. 18, 2009; revised March 9, 2010; accepted April 6, 2010.

This research was supported by Merit Review Funding from the Department of Veterans Affairs (V.L.A.), the New York State Spinal Cord Injury Research Board (V.L.A., J.M.L.), National Institutes of Health Grants 5 R01 NS16996 (L.M.M.) and R01 NS 21998 (J.M.L.), and the Christopher and Dana Reeve Foundation (L.M.M., J.W.F.).

Correspondence should be addressed to Dr. Victor L. Arvanian, Northport Veterans Affairs Medical Center, 79 Middleville Road, Building 62, Northport, NY 11768. E-mail: vlarvanian@notes.cc.sunysb.edu.

DOI:10.1523/JNEUROSCI.4659-09.2010

Copyright $\odot 2010$ the authors $\quad 0270-6474 / 10 / 307761-09 \$ 15.00 / 0$ and Fawcett, 2007). CSPGs consist of a core protein to which is attached one or more chondroitin sulfate glycosaminoglycan side chains. Degrading CSPGs by enzymatic removal of chondroitin sulfate chains with the chondroitin sulfate glycosaminoglycandigesting bacterial enzyme chondroitinase-ABC (Ch'ase) promotes axonal regeneration and stimulates anatomical plasticity in the damaged and undamaged brain and spinal cord and encourages functional recovery (Bradbury et al., 2002; Pizzorusso et al., 2002; Galtrey et al., 2007; Cafferty et al., 2008; Iseda et al., 2008; Kwok et al., 2008; García-Alías et al., 2009).

The goal of this study was to determine whether the elevated level of CSPGs surrounding a spinal cord HX might be involved in the failure of axonal conduction in undamaged axons on the other side of the cord. To answer this question, we designed two sets of experiments. Set 1 was directed toward evaluation of the ability of CSPG degradation to restore axonal conduction that normally declines contralateral to HX. These rats received a HX at midthoracic level with an intraspinal injection of Ch'ase, 6 weeks of behavioral testing, and terminal electrophysiological evaluation of transmission through the HX level or anatomical evaluation of the survival of reticulospinal axons. Set 2 was designed to test the acute effects of CSPGs on axonal conduction in the intact spinal cord. Intact rats received intraspinal injections of purified CSPGs into the thoracic lateral column during the recording procedure, and the effect of these injections on axonal conduction and on monosynaptic transmission to individual lumbar motoneurons was measured.

Our results demonstrate that the postinjury conduction block in axons close to the lesion can be prevented by Ch'ase infusion 
and that NG2, one of the CSPGs upregulated after injury, can block axonal conduction and transmission to motoneurons.

Some of these results have been reported in abstract form (Hunanyan et al., 2009).

\section{Materials and Methods}

These studies were performed on adult $(\sim 210 \mathrm{~g})$ female Sprague Dawley rats in accordance with protocols approved by the Institutional Animal Care and Use Committees at State University of New York (Stony Brook, NY), Veterans Affairs Medical Center (Northport, NY), and Cambridge University (Cambridge, UK).

Surgical procedure for the lateral hemisection and intraspinal injections of Ch'ase at thoracic level T10. Animals were deeply anesthetized with 3\% isoflurane in $100 \% \mathrm{O}_{2}$. Dorsal laminectomy was performed to expose spinal cord segments T9-T11, and HX was performed as previously described (Arvanian et al., 2009). Briefly, one tip of the iridectomy scissors was passed through the entire thickness of the spinal cord dorsal to ventral at the midline, and the left dorsal and ventral columns were cut from lateral to the midline. To assess the effects of CSPG digestion, the rats received two intraspinal injections of either $1 \mu \mathrm{l}$ of $100 \mathrm{U} / \mathrm{ml}$ protease-free Ch'ase (Seikagaku America) or $1 \mu \mathrm{l}$ of $100 \mathrm{U} / \mathrm{ml}$ control enzyme penicillinase (P'ase) (Sigma) immediately after the lesion procedure. Injections were given ipsilateral to the HX $1 \mathrm{~mm}$ lateral to the midline with, the first injection $2 \mathrm{~mm}$ rostral and the second $2 \mathrm{~mm}$ caudal. We used a glass micropipette $(30 \mu \mathrm{m}$ diameter, calibrated for a volume of $1 \mu \mathrm{l}$ ) attached to a $10 \mu \mathrm{l}$ Hamilton syringe connected to a microdrive over a period of $10 \mathrm{~min}$ per injection. In each case, the tip of the injecting pipette was inserted into the cord to a depth of $1 \mathrm{~mm}$. The muscle and skin were closed in layers, and an antibiotic (Baytril, $5 \mathrm{mg} / \mathrm{kg}$, $0.1 \mathrm{ml} \mathrm{s.c.}$ ), an analgesic (Buprenorphine $5 \mathrm{mg} / \mathrm{kg}, 0.1 \mathrm{ml}$ s.c.), and $10 \mathrm{cc}$ of sterile saline were administered subcutaneously.

Behavioral assessment. Rats were allowed to move freely and were scored during a 4 min period for their ability to use their hindlimbs by two independent, blinded observers. Joint movements, paw placement, weight support, and forelimb/hindlimb coordination were judged according to the 21-point Basso-Beattie-Bresnahan (BBB) locomotion scale (Basso et al., 1995). BBB scoring was performed at day 4 and then at weekly intervals for 6 weeks. Additionally, the hindpaws were inked and the animals then walked along a track lined with paper, allowing for the collection of footprints that were then used for gait analysis (García-Alías et al., 2008).

In vivo intracellular and extracellular stimulation and recording in spinal cord. After completing the behavioral evaluation, the rats dedicated for electrophysiological experiments were used to detect conduction in uncut axons passing through the region across from the HX. Rats were deeply anesthetized using a ketamine $(80 \mathrm{mg} / \mathrm{kg}, 0.5 \mathrm{ml}) / \mathrm{xylazine}(10$ $\mathrm{mg} / \mathrm{kg}, 0.5 \mathrm{ml}$ ) mixture intraperitoneally. Heart rate and expired $\mathrm{CO}_{2}$ were monitored continuously. Two dorsal laminectomies of the spinal cord were performed to expose T6-T8 (for placement of the stimulation electrode) and L1-L6 (for placement of recording electrodes at L5). We recorded the responses evoked by ventrolateral funiculus (VLF) stimulation rostral and contralateral to HX in L5 motoneurons recorded intracellularly (Axoprobe amplifier, Molecular Devices) and extracellularly from the L5 ventral horn (P5 amplifier, Grass Instruments) (HX at left T10, recording from right L5 motoneurons, stimulation of right T7 VLF). All signals were digitized at $100 \mathrm{kHz}$, stored on a personal computer, and analyzed off-line using pCLAMP 10.

Motoneurons were impaled with sharp glass microelectrodes $(3 \mathrm{M}$ K-acetate; 50-70 M $\Omega$ resistance) and identified by their antidromic response to stimulation of the cut L5 right ventral root. The resting membrane potential of motoneurons used for analysis ranged from -45 to $-65 \mathrm{mV}$.

For extracellular recording we used a tungsten electrode (resistance: $300 \mathrm{~K} \Omega$ ). The recording tungsten electrode was lowered into the lumbar $\mathrm{L} 5$ spinal cord to a depth of $1.3 \mathrm{~mm}$. It was positioned to enter the cord at the dorsal root entry zone at an angle of $20-22^{\circ}$ from vertical in the sagittal plane (tip directed rostrally).

For electrical stimulation of VLF we used a tungsten electrode inserted in the thoracic cord on the same side as the recording electrodes. To determine an optimal depth for the stimulating electrode, we monitored the evoked response from the extracellular recording electrode positioned in the lumbar ventral horn while inserting the stimulation electrode. The depth of the stimulating electrode from which a maximum response was evoked was usually $\sim 1.7 \mathrm{~mm}$, which is consistent with the location of VLF fibers in the lateral white matter tract. This observation is an electrophysiological confirmation that motoneurons in the lumbar ventral horn receive functional inputs from ipsilateral thoracic VLF, and it is consistent with the results of tracing experiments demonstrating the innervation of lumbar motoneurons from VLF tracts (Reed et al., 2008). After the positions of both extracellular recording and stimulating electrodes were established, we impaled a motoneuron and recorded responses to VLF stimulation. The intensity to evoke a maximum response usually ranged from 300 to $600 \mu \mathrm{A}$ in chronically hemisected cords and $40-100 \mu \mathrm{A}$ in the intact cords (Arvanian et al., 2009). The VLF stimulus had a duration of $50 \mu$ s and was delivered at 0.1 or $1 \mathrm{~Hz}$.

The maximum synaptic response for each cell was determined by examining responses to stimuli of increasing intensity. Peak EPSP amplitude was measured from prestimulus baseline to peak. Maximum responses from all motoneurons (50 consecutive responses per cell) were averaged over all motoneurons recorded in each rat before and after CSPG injections, respectively. For extracellularly evoked responses, the responses to 500 consecutive stimuli were averaged and we measured the peak-to-peak value of the action potential volley (Fig. $1 B$ ) as well as the synaptic response.

Intraspinal injections of CSPGs. Rats were anesthetized as described above, and dorsal laminectomy of the spinal cord was performed to expose T6-T8 (for placement of stimulation electrode in T7), T10 (for placement of injection capillary), and L1-L6 (for placement of recording electrodes in L5). Intracellular and extracellular recordings were performed as described above. After recording from 7-10 different motoneurons in each untreated spinal cord, we injected CSPG, specifically the extracellular domain of NG2 (Uhgrin et al., 2003), neurocan (Millipore), and aggrecan (Sigma), into the lateral column of thoracic cord between the stimulation and recording sites (unless otherwise specified). The injection electrode was positioned in the T10 lateral white matter of the spinal cord using a micromanipulator to ensure that the injections were made in the vicinity of axons that were being stimulated. The position (lateral to midline) and depth of the tip of the injecting electrode were determined by position and depth (plus $100 \mu \mathrm{m}$ to account for the greater thickness of the cord in T10) of the stimulating electrode (at T7). After injection of CSPGs, we usually recorded from the same motoneuron for up to $40 \mathrm{~min}$, and after that we recorded EPSPs from 7-10 additional motoneurons. The extracellular response was recorded continually before and after CSPG administration. We compared the average change in responses before and after CSPG and averaged the change over all such animals.

Tracing experiments to evaluate density of fibers that may form the stronger projections in Ch'ase-treated spinal cord. The rats dedicated for anatomical study were anesthetized as described above and injected with the tracer biotinylated dextran amine (BDA) into the right reticular formation using stereotaxic coordinates. After $14 \mathrm{~d}$, transcardical perfusion was performed and a block of T5-L2 spinal segments was used to prepare $20-\mu \mathrm{m}$-thick longitudinal sections cut on a cryostat. Sections were processed as described previously (Galtrey et al., 2007; García-Alías et al., 2008), and BDA-traced reticulospinal axons were counted in the right white matter contralateral to the injury. The number of labeled axons 1 $\mathrm{cm}$ caudal and $1 \mathrm{~cm}$ rostral to the segment across from the HX was expressed as a ratio and compared between penicillinase- and Ch'asetreated preparations using one-way ANOVA for statistical comparisons.

Immunofluorescence double staining for NG2 and CASPR and confocal microscopy to evaluate distribution of NG2 after its intraspinal injections. Rats were perfused with $400 \mathrm{ml}$ of $4 \%$ paraformaldehyde in $0.1 \mathrm{M}$ PBS containing $5 \%$ sucrose either 15 or 60 min after injection of NG2. The spinal cord was removed immediately and postfixed for $2 \mathrm{~h}$. A segment of spinal cord containing the NG2 injection site was cryoprotected in $30 \%$ sucrose, frozen, sectioned at $20 \mu \mathrm{m}$ on a cryostat, and collected serially onto gelatin-coated slides. Immunofluorescence staining of injected spinal cords with rabbit anti-NG2 (Millipore Bioscience Research Reagents) 
Evoked EPSPs (intracellular) Evoked potentials (extracellular)
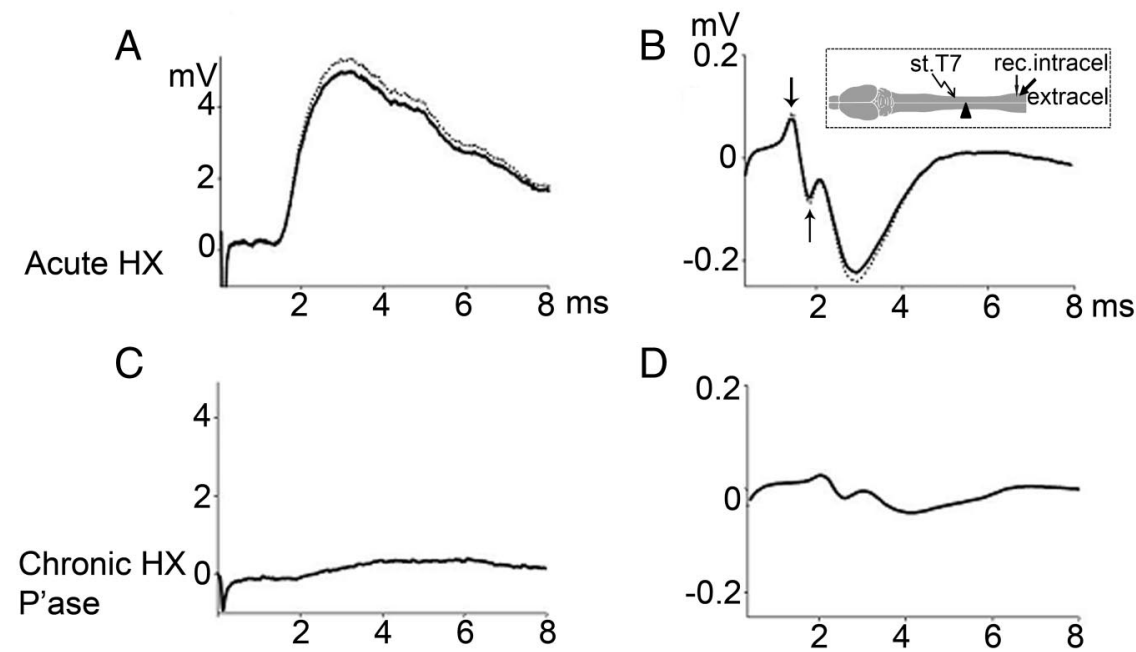

$\mathrm{D}$

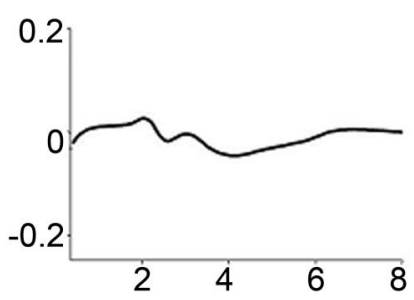

F

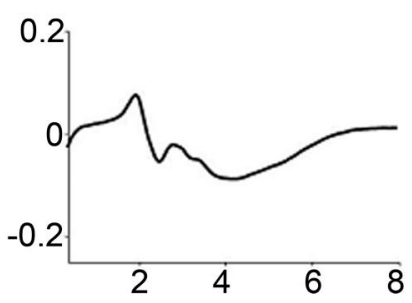

Figure 1. Representative traces of EPSPs [intracellular (intracel)] $(\boldsymbol{A}, \boldsymbol{C}, \boldsymbol{E})$ and evoked potentials [extracellular (extracel)] ( $\boldsymbol{B}, \boldsymbol{D}, \boldsymbol{F})$ under the following conditions: before (dotted line traces) and after (solid line traces) acute $\mathrm{HX}(\boldsymbol{A}, \boldsymbol{B})$; chronic $\mathrm{HX}$ and P'ase treatment $(\boldsymbol{C}, \boldsymbol{D})$; chronic $\mathrm{HX}$ and Ch'ase treatment $(\boldsymbol{E}, \boldsymbol{F})$. Inset $(\boldsymbol{B})$ shows position of stimulation (st)/recording (rec.) electrodes with respect to HX. The extracellular responses consist of two components: the early biphasic arriving volley (measured between the arrows) and the later downward extracellular synaptic response (measured from baseline to peak). Note that P'ase did not reverse the effects of chronic hemisection in attenuating the contralateral volley, whereas Ch'ase did partially attenuate it (see Results).

was performed as described previously (Tan et al., 2006). Sections were incubated overnight in a mixture of rabbit anti-NG2 (1:500) and mouse anti-CASPR (1:1000; Neuromab, University of California, Davis, CA) at $4^{\circ} \mathrm{C}$, washed in three changes of PBS, and then incubated in goat antirabbit and goat anti-mouse Alexa Fluor-conjugated secondary antibodies at a 1:1000 dilution for $1 \mathrm{~h}$. Following three rinses in PBS and a brief rinse in $\mathrm{dH}_{2} 0$, slides were coverslipped with Fluoromount-G (Southern Biotech). Z-stack images were taken with a Zeiss 510 confocal microscope as described previously (Tan et al., 2006).

Statistics. Behavioral testing: the scores were compared between animals treated with P'ase and Ch'ase at different time points after the injury using two-way repetitive measures ANOVA for statistical comparisons, followed by a Tukey test. For tracing, the number of labeled axons was compared between penicillinase- and Ch'ase-treated preparations using one-way ANOVA (Tukey test). For electrophysiology, the peak amplitudes of EPSPs and the composite potentials averaged over all cells in each rat were compared using one-way ANOVA ( $n=$ number of rats); if significant differences were observed between groups, a Student-Newman-Keuls test was used for pairwise comparisons. For statistical data analyses we used SigmaPlot 11.0 software. Means are expressed \pm SEM.

\section{Results}

Set 1: Ch'ase infusion prevents conduction failure in surviving axons and facilitates recovery of function after chronic hemisection

This set of experiments was designed to study whether treatment of the hemisected spinal cord with Ch'ase immediately after HX prevents or reverses the failure of conduction contralateral to HX and facilitates recovery of function during the chronic stage of injury. Rats received a HX at T10 and an immediate perilesional, intraspinal injection of Ch'ase or the neutral bacterial enzyme P'ase as control. Following 6 weeks of behavioral testing, one subgroup was used for electrophysiological evaluation of transmission through the HX to motoneurons below the HX; the other subgroup was injected with an anatomical tracer to evaluate the survival of reticulospinal axons.

\section{Acute hemisection does not affect monosynaptic transmission to lumbar motoneurons and conduction in contralateral axons}

We recorded in vivo the monosynaptic EPSP in motoneurons intracellularly and the field potentials extracellularly from the right L5 ventral horn in response to electrical stimulation of the ipsilateral VLF at T7; Figure 1 shows position of electrodes. We measured these monosynaptic responses before and immediately after (within $10 \mathrm{~min}$, and then during next $2 \mathrm{~h}$ ) lateral HX of the left thoracic cord at the T10 level (Fig. 1). In uninjured rats, the peak amplitude of the maximum monosynaptic EPSP (recorded intracellularly) was $5.2 \pm 0.7$ $\mathrm{mV}$, and its latency (measured from stimulus artifact to response onset) was $1.3 \pm 0.2 \mathrm{~ms}$ (Fig. 1 A). The extracellular response consisted of an early biphasic component representing the volley of action potentials (Fig. $1 \mathrm{~B}$, measured between the arrows) averaging $0.27 \pm 0.1 \mathrm{mV}$, and a later component representing the synaptic response averaging $0.3 \pm 0.1$ $\mathrm{mV}(n=5)$. After the contralateral left thoracic cord was hemisected at T10, the peak amplitude and the latency of intracellular responses $(4.6 \pm 0.8 \mathrm{mV} ; 1.3 \pm 0.2 \mathrm{~ms})$, as well as the peak amplitude of extracellular responses $(0.25 \pm 0.1 \mathrm{mV}$ early extracellular, $0.26 \pm 0.2 \mathrm{mV}$ later extracellular), did not change significantly ( $p=0.1, n=5)$ (Fig. $1 A, B)$.

These results demonstrate that monosynaptic responses in lumbar motoneurons recorded from the right-side ventral horn and evoked by stimulation of the right-side VLF are unaffected by acute HX of the left side of the cord. These results are consistent with recent results that demonstrated no significant effect of acute left HX on the polysynaptic responses from right VLF above the hemisection to contralateral motoneurons below the level of the HX (Arvanian et al., 2009).

\section{Chronic hemisection leads to conduction block in contralateral axons}

In a previous study we showed that spinal cord hemisection leads to a gradual onset of conduction block in axons contralateral to the lesion, starting at $1-2$ weeks and continuing for at least 14 weeks (Arvanian et al., 2009). We repeated these experiments with the addition of a control infusion of penicillinase, a bacterial enzyme with no effect in mammals delivered to the spinal cord parenchyma on either side of the lesion at the time of operation $(n=5)$. After 6 weeks, rats were assessed electrophysiologically 
by in vivo intracellular recording from individual motoneurons and extracellular recording from the ventral horn at the lumbar L5 segment (Fig. 1). In these rats, we found a marked reduction of the action potential volley and the synaptic responses evoked by stimulation of VLF above the HX level and recorded below. The mean peak amplitude of each response recorded from the chronically hemisected and P'ase-treated rats was significantly smaller (intracellular EPSP peak amplitude, $0.7 \pm 0.3 \mathrm{mV}$; extracellular early peak, $0.04 \pm 0.02 \mathrm{mV}$; and later peak, $0.05 \pm 0.02 \mathrm{mV}$ ) (Fig. $1 C, D)$ compared with acutely hemisected rats $(p=<0.001$ ) (Fig. $1 A, B)$. The latency of intracellular responses in hemisected and P'ase-treated rats was significantly longer compared with that of noninjured controls $(2.2 \pm$ $0.3 \mathrm{~ms} ; p=0.03$ ). Similar responses measured from chronically hemisected rats treated with P'ase were not significantly different from those recorded previously (Arvanian et al., 2009) in rats that received HX and no treatment for 6 weeks (both peak amplitude and latency; $p=0.1, n=5)$.

\section{Intraspinal Ch'ase attenuates conduction block but does not affect conduction velocity in axons adjacent to chronic spinal hemisection}

We next asked whether the decline in axonal conduction and synaptic responses that developed during the course of chronic HX (Arvanian et al., 2009) would still be observed if intraspinal injections of Ch'ase were administered at the time of HX. To estimate effects of Ch'ase on axonal conduction, we measured the amplitude of action potential volleys and EPSPs. In addition, to estimate the effects of Ch'ase on conduction velocity, we measured the latency of these responses. Immediately after HX, rats were injected intraspinally with Ch'ase $(n=9)$ and allowed to survive for 6 weeks of behavioral assessment; they were then prepared for either electrophysiological recordings $(n=6)$ or anatomical tracing $(n=3)$. The peak amplitude of all responses measured from rats of the Ch'ase group $(n=5)$ was markedly larger compared with that of the P'ase group described above; intracellular: EPSP peak amplitude, $2.2 \pm 0.4 \mathrm{mV}$ Ch'ase (vs $0.7 \pm 0.3 \mathrm{mV}$ P'ase), $p=<0.001$ (Fig.1C,E); extracellular early peak: $0.15 \pm 0.03 \mathrm{mV}$ Ch'ase (vs $0.04 \pm 0.02 \mathrm{mV}$ P'ase), $p=<0.001$; later peak: $0.14 \pm 0.04 \mathrm{mV}$ Ch'ase (vs $0.05 \pm 0.02 \mathrm{mV}$ P'ase), $p=0.01$ (Figure $1 D, F$ ). These effects of Ch'ase on the amplitude of the action potential volleys and EPSPs indicate that Ch'ase treatment either partly prevents or reverses the decline in action potential conduction through VLF white matter contralateral to a chronic HX. However, this action of Ch'ase was not associated with changes in the latency of intracellular responses $(2.1 \pm 0.2 \mathrm{~ms}$ in Ch'ase group vs $2.2 \pm 0.3$ $\mathrm{ms}$ in P'ase group, $p=0.2$ ). Thus, Ch'ase attenuated the conduction block in axons adjunct to HX, but this action was not associated with changes in conduction velocity.
After completion of these recordings, rats were transcardially perfused, and $10-\mu \mathrm{m}$-thick, cresyl violet-stained cross-sections were cut for reconstruction of the injury site, as described previously (Arvanian et al., 2009). We found that most injuries were uniform with a complete lesion of the left side of the cord (with the exception of one case, which was excluded from the analysis).

\section{Ch'ase has no effect on number (density) of unlesioned axons contralateral to hemisection}

To determine whether the physiological effects of Ch'ase on the conduction of axons contralateral to HX could be associated with the changes in number of reticulospinal axons, the tracer BDA was injected into the right reticular formation, and longitudinal sections were used to quantify the reticulospinal axons in the right white matter contralateral to the injury (Fig. $2 A-G$ ). We computed the ratio of the labeled axons counted $1 \mathrm{~cm}$ above vs 1 $\mathrm{cm}$ below the HX in all consecutive sections for each rat. No difference in the preservation of these axons across from the injury was found between P'ase $(n=3)$ and Ch'ase $(n=3)$ groups $(p=0.4)$ (Fig. 2D).

\section{Ch'ase treatment improves locomotor behavior}

The animal's ground locomotion performance was evaluated using the open field BBB score and gait analysis. Consistent with the 
Evoked EPSPs (intracellular)

A
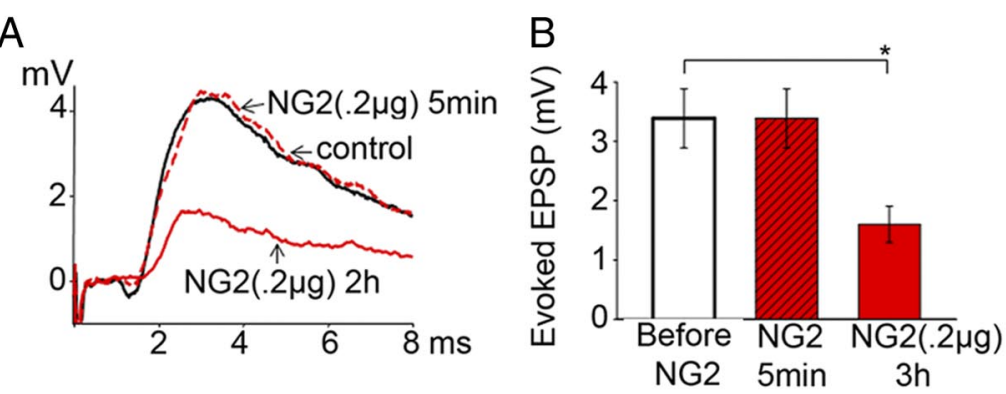

Evoked potentials (extracellular)

C

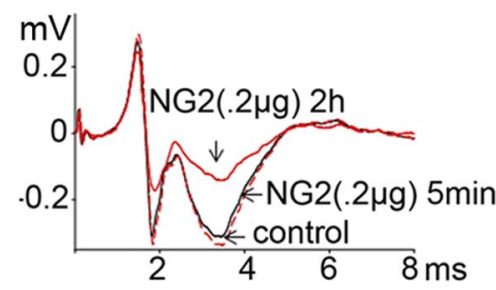

D

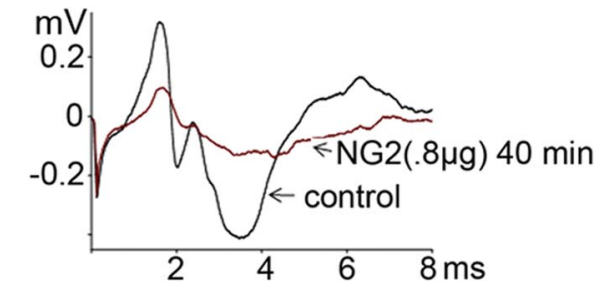

E

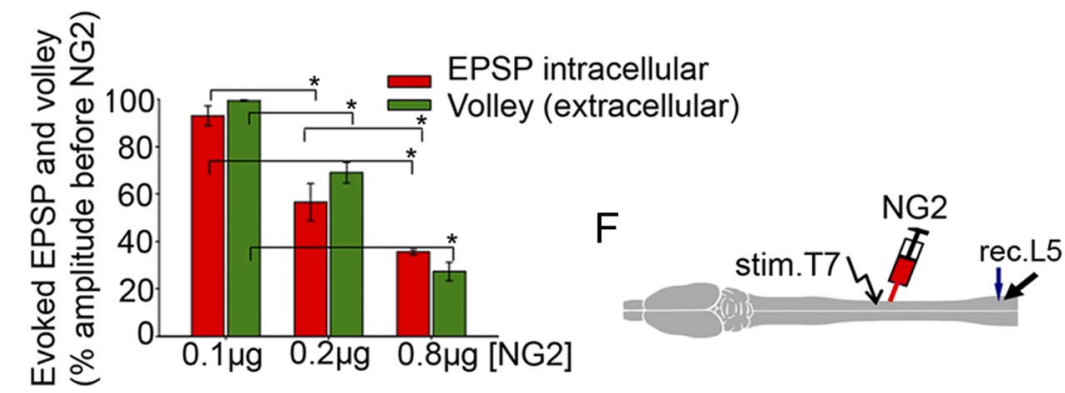

Figure 3. Effects of intraspinal NG2 injections in lateral T10 on the evoked EPSP (intracellular) and the evoked composite potentials (extracellular). $\boldsymbol{A}$, Superimposed averaged responses: control EPSP (black); 5 min after NG2 injection (dotted red); $3 \mathrm{~h}$ after NG2 injections (solid red). B, Summary of results demonstrating delayed depression of evoked EPSPs after NG2. All means represent peak amplitude of the responses from 7 rats, and for each rat the response was an average of maximum responses recorded intracellularly from 7-10 motoneurons before, 5 min after, and $3 \mathrm{~h}$ after injections of NG2 $(0.2 \mu \mathrm{g})$. C, Evoked composite potentials in the same rat under the same conditions as in $\boldsymbol{A}$ to show depression of both action potential volley and synaptic components of evoked response after $0.2 \mu \mathrm{g}$ of NG2. D, Higher dose of NG2 $(0.8 \mu \mathrm{g})$ induces greater depression of the action potential volley and synaptic components of evoked composite responses; averaged responses recorded before (black) and $40 \mathrm{~min}$ after $0.8 \mu \mathrm{g} \mathrm{NG2}$ injection (dark red). $E$, Summary of results demonstrating dose-dependent action of NG2 on EPSPs (intracellular, red columns) and action potential volley (extracellular, green columns). Ordinate represents mean ratio of the peak amplitudes of the maximum responses from 5-7 rats (7-10 motoneurons in each rat) before and $3 \mathrm{~h}$ after NG2 injections at denoted doses. $\boldsymbol{F}$, Diagram to show the position of the stimulation (stim.)/recording(rec.)/injection electrodes. Asterisk $\left(^{*}\right)$ above the brackets $(\boldsymbol{B}, \boldsymbol{E})$ represents significant difference between the corresponding bars $(\boldsymbol{B}, p=0.01 ; \boldsymbol{E}, p=<0.001)$.

previously described locomotor performance of rats that received lateral $\mathrm{HX}$ of the thoracic cord and no treatment (Arvanian et al., 2009), the animals in both the P'ase and the Ch'ase groups spontaneously recover locomotor function, but the recovery process slows at $\sim 2$ weeks post-HX (i.e., the time point when the conduction of unlesioned axons contralateral to HX becomes blocked) (Fig. 2). The animals treated with Ch'ase, however, showed better interlimb coordination as reflected in their higher $\mathrm{BBB}$ scores, but the difference was significant only on day 42 post-HX, the longest time tested in the open field. (Fig. $2 \mathrm{H}, \mathrm{I}$ ). Consistent with the enhanced motor performance and better body stability observed using $\mathrm{BBB}$, gait analyses revealed a more normal base of support of the hindlimbs in the Ch'ase group (Fig. $2 \mathrm{~J}$ ).

In summary, the results of anatomical tracing suggest that Ch'ase did not alter the number of reticulospinal axons across the chronic HX, but electrophysiological data demonstrated that the treatment partly prevented loss of conduction in these fibers. The rescue of axonal conduction has a timing appropriate to contribute to the improved locomotor performance in Ch'ase-treated animals.

\section{Set 2: Acute effects of purified CSPGs on conduction and synaptic transmission in the intact spinal cord} The data presented above suggest that the postlesion decline in conduction in the intact VLF could be due to an accumulation of CSPGs around the lesion site. To test directly whether increased levels of CSPGs alter axonal conduction and the synaptic response, we injected purified CSPGs into the intact spinal cord at T10 and recorded responses in the L5 ventral horn elicited by stimulating VLF at T7. We compared responses obtained just before, immediately after, and 2-3 h after the injection.

\section{In vivo intraspinal injections of known pharmacological agents induce the predicted changes in both axonal conduction and postsynaptic potentials recorded in vivo from the adult rat spinal cord}

To evaluate whether intraspinal injection is an effective delivery method with preservation of continuous, long-lasting, in vivo electrophysiological recordings, we injected the following control substances directly into the spinal cord: saline, 6-cyano7-nitroquinoxaline-2,3-dione (CNQX), an AMPA/kainate receptor blocker that selectively blocks the initial monosynaptic response of motoneurons (Arvanian and Mendell, 2001), or tetrodotoxin (TTX), an inhibitor of voltage-gated $\mathrm{Na}$-channels, known to diminish propagation of the action potential.

Injections of saline $(1-3 \mu \mathrm{l})$ either in the $\mathrm{L} 5$ ventral horn close to the recording area or in the T10 lateral white matter had no effect on the evoked EPSP (intracellular) or composite responses (extracellular) (supplemental. Fig. $1 A, B$ ). Intraspinal injections of CNQX [200 $\mu \mathrm{M}$ (concentration in pipette) $/ 0.8 \mu \mathrm{l}$ (injected volume)] close to the recording area depressed the intracellularly recorded EPSP, beginning $\sim 4$ min postinjection. In the case of the composite extracellular responses, the CNQX injections depressed only the later synaptic component of the evoked response, while the earlier action potential volley was not affected (supplemental Fig. 1, available at www.jneurosci.org as supplemental material, $n=3)$. Five minutes after injecting TTX $(30 \mu \mathrm{M} / 0.8 \mu \mathrm{l})$ into T10 lateral white matter, all responses recorded intracellularly and extracellularly at L5 in response to stimulation at T7 were blocked $(n=5)$ (supplemental Fig. 1, available at www.jneurosci.org as supplemental material). 


\section{Injection of NG2 in T10 white matter causes depression of EPSPs recorded from L5 motoneurons in response to stimulation of T7 VLF}

Intraspinal injections of NG2 into the T10 lateral white matter of uninjured rats between stimulating and recording electrodes (Fig. 3F), consistent with the level of chronic $\mathrm{HX}$ in the Ch'ase experiments (Fig. 1), depressed the EPSP responses recorded intracellularly from lumbar motoneurons in a time- and dose-dependent manner (Fig. 3A,E). At relatively low doses $(0.1 \mu \mathrm{g} / 0.8 \mu \mathrm{l}, n=5 \mathrm{rats}), \mathrm{NG} 2 \mathrm{had}$ no significant action (Fig. 3E). Immediately after injection of NG2 at a higher dose $(0.2 \mu \mathrm{g} / 0.8 \mu \mathrm{l}, n=7 \mathrm{rats})$, the amplitude of the evoked EPSPs was not changed, suggesting that the injection procedure did not damage conducting axons and the recorded cells. However, 1.5$2.5 \mathrm{~h}$ after injection of $0.2 \mu \mathrm{g}$ of NG2 into the T10 white matter, the amplitude of the EPSPs recorded intracellularly from L5 motoneurons was significantly depressed $((p=0.03)$ Fig. 3E). These NG2-induced changes in EPSP peak amplitude were not associated with the changes in latency of these intracellular responses $(1.3 \pm 0.2 \mathrm{~ms}$ before and $1.4 \pm 0.2 \mathrm{~ms}$ after NG2, $p=$ $0.5)$. Injection of the higher doses of NG2 $(0.8 \mu \mathrm{g} / 0.8 \mu \mathrm{l}, n=5$ rats $)$ into T10 induced a greater depression of the evoked EPSPs in L5 motoneurons, and this depression occurred more rapidly, i.e., all measured EPSP responses reached the lower steady-state level within $\sim 30-45$ min after the injection (Fig. $3 D, E$ ).

\section{Injection of NG2 causes axonal conduction block}

The most likely explanation for the depression of EPSPs in L5 motoneurons induced by intraspinal injections of NG2 between the recording and stimulating electrodes (Fig. $3 A, B$ ) is failure of the action potential to conduct past the injection site. Therefore, in addition to intracellular recordings, we performed simultaneous extracellular recordings from the L5 ventral horn and measured the effects of intraspinal injections of NG2 on the action potential volleys (Fig. 3C,D). We found that NG2 injected into T10 white matter between the stimulating and recording electrodes reduced the amplitude of the extracellular volley, which closely paralleled the changes in the amplitude of intracellular EPSPs described above, with a similar pattern for the time- and dose-dependent depression induced by NG2 (Fig. 3E).

\section{Effects of NG2 depended on the level of intraspinal injections} vs position of the stimulating electrode

To demonstrate that NG2 injections were effective only at points between the stimulating and recording electrode but ineffective if placed outside the conduction path, we injected NG2 into either L4-L5 (near the recording electrode positioned in L5) or T7 (just rostral to stimulating electrode) (Fig. 4). Injections of NG2 (0.2 $\mu \mathrm{g}, n=5$ ) between the stimulating and the recording electrodes, but near the recording area (L5), induced a degree of depression of the intracellular EPSPs $(48.0 \pm 10.8 \%, p=0.008, n=4)$ and extracellular composite responses $(70.0 \pm 13.9 \%, p=0.04, n=$ 4), similar to those detected in the case of NG2 injections at T10 (Fig. 4A). No effects were observed in response to injections of NG2 at T7, i.e., above the stimulating electrode ( $p=0.6, n=3)$ (Fig. 4B). These results suggest that NG2 depresses conduction at the level of the injections.

\section{Injection of aggrecan or neurocan does not cause conduction block}

We next asked whether the ability to block conduction was a specific property of NG2 or a general property of CSPGs that are present at CNS injury site. We performed intraspinal injections of two other CSPGs, aggrecan and neurocan, and performed similar experiments. In contrast to the inhibitory action of NG2 injected in T10 between stimulating and recording electrodes (Fig. $3 F)$, similar intraspinal injections of either aggrecan $(0.8 \mu \mathrm{g}$, $p=0.3, n=5)($ Fig. $5 A, B)$ or neurocan $(0.8 \mu \mathrm{g}, p=0.6, n=4)$ had no significant effect on the evoked responses recorded either 

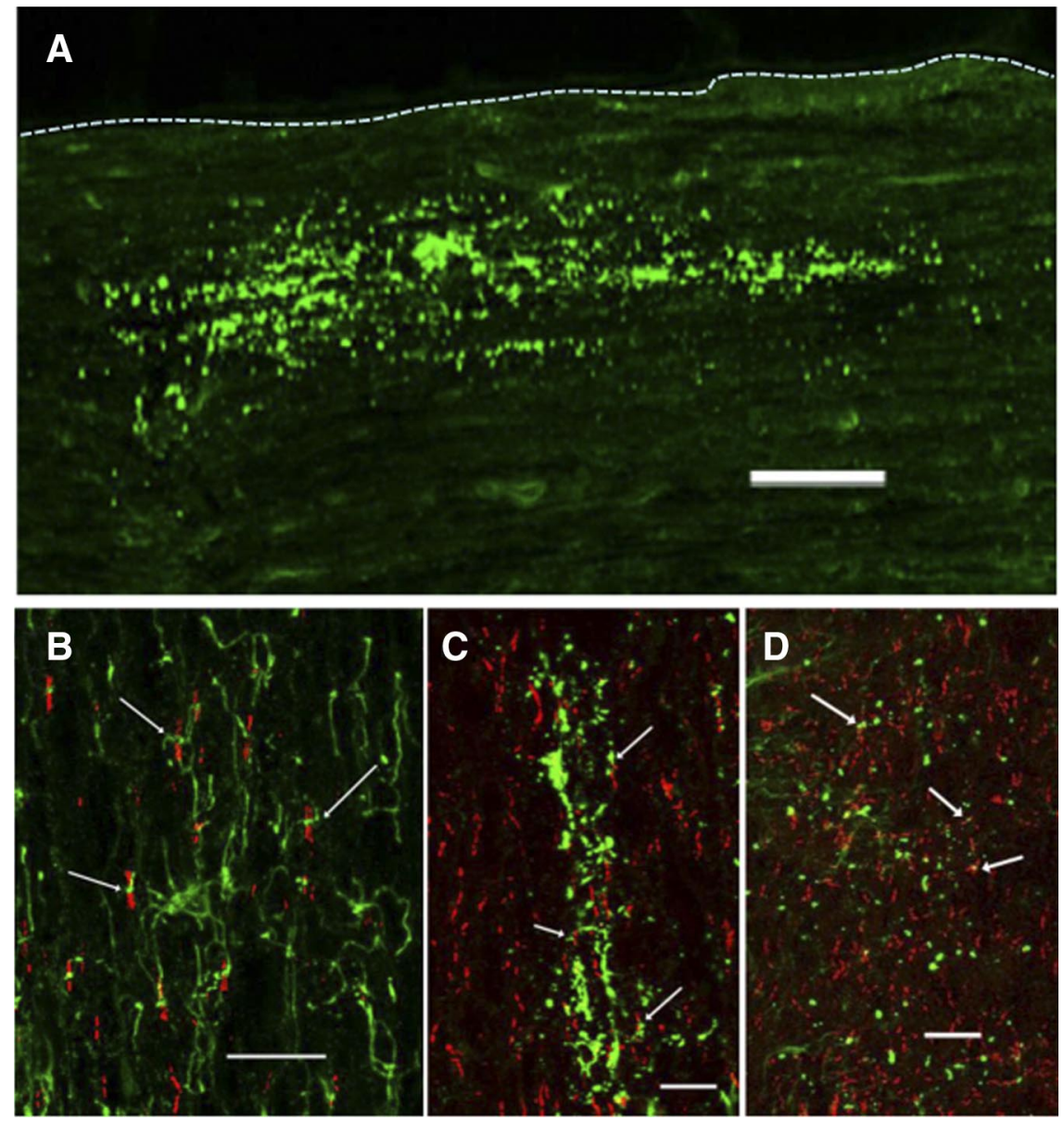

Figure 6. Localization of endogenous and exogenous NG2 in the rat spinal cord. Adult rats were injected with NG2 $(1 \mu \mathrm{g})$ and killed 15 or 60 min later. $A$, Overview of the distribution of exogenous NG2 $15 \mathrm{~min}$ after injection. The injected protein distributes over a wide area in a linear pattern, suggesting an association with axons. Dotted line outlines the lateral edge of the cord. Scale bar, $100 \mu \mathrm{m}$. B. Appearance of NG2-positive oligodendrocyte progenitor cells (green) and CASPR (red) in the spinal cord contralateral to the injection site. The arrows point to areas were NG2-postive processes make close contact with nodes of Ranvier. $C, \boldsymbol{D}$, Exogenous NG2 $15 \mathrm{~min}(\boldsymbol{C})$ and $60 \mathrm{~min}(\boldsymbol{D})$ after injection. The protein is distributed in a linear fashion and the arrows point to NG2 accumulations close to CASPR-positive nodes of Ranvier. Scale bars: $\boldsymbol{B}-\boldsymbol{D}, 20 \mu \mathrm{m}$.

intracellularly or extracellularly. In rats tested initially on one side with aggrecan with no decline in conduction observed, subsequent injections of NG2 in the contralateral white matter depressed the evoked responses on that side (Fig. 5C,D) $(n=3)$. These results suggest that the depression of axonal conduction is specific to NG2 but not to the other CSPGs studied, i.e., aggrecan or neurocan.

\section{Localization of endogenous and exogenous NG2 in the rat spinal cord}

To study the localization of the injected NG2, four adult rats were injected with NG2 $(1 \mu \mathrm{g})$, perfused with fixative 15 or $60 \mathrm{~min}$ later, and double stained for NG2 and CASPR (a marker of the nodes of Ranvier) (Fig. 6). Figure 6A shows that the injected NG2 distributes over a wide area $(>500 \mu \mathrm{m}$ along the rostral-caudal axis) in a linear pattern suggesting an association with axons. In the spinal cord contralateral to the injection site, the processes of endogenous NG2-positive oligodendrocyte progenitor cells (green) make close contact with some nodes of Ranvier (red) (Fig. 6B, arrows). At the injection site $15 \mathrm{~min}$ after the injection, exogenous NG2 appears as puncta distributed in a linear fashion, presumptively along axons. Many of the NG2 puncta accumulated close to CASPRpositive nodes of Ranvier (Fig. $6 C, D$ ). At 60 min postinjection, the size and number of puncta was reduced but were still closely associ- ated with CASPR-positive nodes of Ranvier (Fig. 6D). Nodes of Ranvier are directly involved in propagation of action potentials along the axons, including the spinal cord axons (Wang et al., 1997; Black et al., 2006). Our results suggest that NG2 may have a close interaction with nodes of Ranvier and, thus, changes in the level of NG2 may influence, i.e., depress conduction in these axons.

To compare the distribution of endogenous NG2 in the vicinity of hemisection lesion, six adult rats received a HX injury. Three rats were perfused $24 \mathrm{~h}$ post-HX, when conduction through contralateral to HX side is close to normal, and three rats were perfused $12 \mathrm{~d}$ post-HX, when conduction across from $\mathrm{HX}$ is diminished (Arvanian et al., 2009). Spinal cords from both groups were simultaneously stained for NG2 as described in Materials and Methods. As shown in supplemental Figure 2 (available at www.jneurosci.org as supplemental material), there is upregulation of NG2 signal around the HX and clusters of increased NG2 levels in the white matter contralateral to HX in all three 12 day post-HX animals. In contrast in all $24 \mathrm{~h}$ post-HX rats, the level of NG2 staining around HX was relatively low and we did not observe clusters of NG2 in white matter across from HX.

\section{Discussion}

The major finding of this study is that CSPGs, either upregulated after spinal cord injury or directly injected, are able to block axonal conduction in intact axons. We found that administration of Ch'ase across from the injured region at the time of HX largely prevented (or reversed) the decline of conduction observed after 6 weeks in untreated preparations (Arvanian et al., 2009) or after injections of control P'ase (Fig. 1). We also found that NG2, a proteoglycan known to be released into the zone of injury (Jones et al., 2002; Leonard et al., 2003), acutely diminishes impulse conduction (Fig. 3).

NG2 has been suggested as a major chondroitin sulfate proteoglycan produced after spinal cord injury (Jones et al., 2002). Moreover, the levels of NG2 as well as neurocan and brevican have been found to be upregulated by $2 \mathrm{~d}$ and remain elevated for at least 3 weeks following partial spinal cord injuries (Tang et al. 2003; Massey et al., 2008). Here. we found that Ch'ase partially reversed the effects of $\mathrm{HX}$ when studied 6 weeks after the injury. We have previously shown that the conduction decrement begins as early as 1 week after the injury and continues for several weeks (Arvanian et al., 2009). It has previously been shown that Ch'ase injected into the CNS retains some activity for $\sim 2$ weeks (Lin et al., 2008), so the treatment may have prevented the accumulation of glycanated CSPGs during the period when conduction block is first seen, and this action is sufficient to maintain axon conduction even after the enzyme is no longer present. Further studies using multiple Ch'ase administration should be undertaken to better understand the time course of this mechanism. 
In previous experiments, the improved neurological function displayed by spinally injured animals treated with Ch'ase has been ascribed to enhanced axon regeneration and sprouting (Bradbury et al., 2002; Houle et al., 2006; Busch and Silver, 2007; Galtrey et al., 2007; García-Alías et al., 2008). In the present experiments using an HX model with incomplete lesion, where fibers contralateral to HX were intact, Ch'ase treatment also led to improved motor function with enhanced locomotion during the chronic phase. We previously showed that the spontaneous recovery of locomotor function after HX levels off during the chronic stage, as conduction deficits develop in the intact contralateral white matter (Arvanian et al., 2009). Here, we demonstrate slightly better behavioral recovery in HX rats treated with Ch'ase, beyond the partial spontaneous recovery of function after hemisection injury and P'ase treatment. It will be important to determine whether this difference observed $42 \mathrm{~d}$ after HX is permanent and, if not, how long it persists.

To elucidate the role of CSPGs on these changes in axonal conduction and to discover whether these effects are general to all CSPGs or specific to one, we injected three purified CSPGs, NG2, aggrecan, and neurocan into the lateral column of uninjured thoracic cords. NG2 and neurocan are upregulated after spinal cord injury (Jones et al., 2002; Leonard et al., 2003), whereas aggrecan exhibits complex changes (Schwartz et al., 1996; Lemons et al., 2001; Teng et al., 2008). We found that intraspinal injection of NG2 $(0.2-0.8 \mu \mathrm{g})$ into the intact spinal cord depressed both the early (action potential volley) and later (synaptic) components of evoked potentials measured in ventral horn only when injected between the stimulating and recording electrodes (Fig. 3). In contrast, aggrecan and neurocan had no significant effect on impulse conduction. Intracellular recordings from motoneurons to which fibers in VLF project revealed that injection of NG2 results in a decline in the amplitude of the synaptic response in target motoneurons. The depression of the action potential volley strongly suggests that the depression of synaptic responses is the result of conduction block at the level of NG2 injection.

CPSGs have not previously been implicated in axonal conduction block. How might such a block come about? Failure of conduction in large myelinated axons, such as reticulospinal axons that form monosynaptic connections to motoneurons, could indicate an event at the nodes of Ranvier, probably ultimately affecting the function of voltage-gated sodium channels. CSPGs are found diffusely in the extracellular matrix throughout the undamaged adult CNS, but also in a condensed matrix around neurons as in perineuronal nets and in similar structures at nodes of Ranvier (Melendez-Vasquez et al., 2005; Kwok et al., 2008). Many nodes of Ranvier in the CNS are contacted by an NG2-positive glial process, and NG2 accumulates within the extracellular matrix at peripheral nodes of Ranvier (Butt et al., 1999; Martin et al., 2001). Moreover, two other CSPGs are found at CNS nodes and appear to play a part in their construction (Butt et al., 2002; Melendez-Vasquez et al., 2005; Bekku et al., 2009; Dours-Zimmermann et al., 2009). The functions of NG2 and other CSPGs found at nodes of Ranvier are unknown, but it is likely that they participate in either the construction or stabilization of nodal specializations such as partitioning ion channels into discrete structural domains.

Ch'ase is known to cause degradation of CSPGs by removing chondroitin sulfate glycosaminoglycan (GAG) side chains. Therefore the Ch'ase-induced prevention of the conduction blockade in hemisected rats may be the result of the removal of GAG chains from NG2. Although the GAG chains of NG2 may not be required for axon growth inhibition in vitro (Dou and Levine, 1994), recent data also suggest that chondroitin sulfate GAGs can be important in cer- tain functions of NG2 (Uhgrin et al., 2003, Laabs et al., 2007). The role of NG2 and its associated chondroitin 4-sulfate GAG chains in axonal conduction needs further investigation.

The inhibitory effect of NG2 on conduction in uninjured cord appears to mimic the block of axon conduction that was observed previously contralateral to chronic spinal cord hemisection. However, conduction deficits induced by chronic HX injury in VLF axons were recently found to be associated with partial demyelination of fibers in the VLF across from the HX, which was considered to account for the reduced conduction velocity of fibers in this region (Arvanian et al., 2009). The present results show that the partial recovery of conduction deficits in Ch'asetreated rats is characterized by recovery in the amplitude of the evoked responses in the lumbar cord, but no significant change in their latency. Thus, no change in conduction velocity of the axons could be inferred. Consistent with these effects of Ch'ase in injured cords, administration of NG2 to the intact cord induced the blockade in conduction, but no changes in conduction velocity. Therefore, we speculate that two mechanisms may be operating contralateral to lateral hemisection, fiber demyelination (Arvanian et al., 2009) and impulse blockade at nodes of Ranvier. Both of these would contribute to the decline of conduction observed after HX. After administration of NG2 to the intact cord, the blockade in conduction is caused selectively by nodal dysfunction. The recovery in HX animals after Ch'ase would occur by reversal of nodal dysfunction and not the demyelination.

We speculate that one possible explanation for the finding that NG2, but not neurocan or aggrecan, depressed axonal conduction is the small size of the NG2 with side-chain complex in comparison with other two CSPGs (Schwartz et al., 1996; Tang et al., 2003; Galtrey and Fawcett, 2007). Its small size would allow NG2 to enter the intranodal space with resulting functional consequences. These questions require further investigation. Our results suggest that one mechanism underlying the decline of conduction induced by chronic hemisection is upregulation of NG2 around the injury site in close association with nodes of Ranvier, and that this molecule in some way affects the ability of the nodes to sustain action potential conduction.

Although the accumulation of CSPGs in and around injury site is thought to be a major barrier for axonal regeneration and recovery of function after SCI in the adult mammalian spinal cord (Lemons et al., 1999; Davies et al., 2004; García-Alías et al., 2008), increasing evidence suggests a possible beneficial role for CSPGs in the scar tissue as part of the endogenous local immune regulation and repair process (Rolls et al., 2009). CSPGs are involved in a variety of CNS functions, including the modulation of cell adhesion, cell migration, axonal outgrowth, and synapse formation (Schwartz et al., 1996; Yamaguchi, 2000; Paukert and Bergles, 2006; Wigley et al., 2007; Kukley et al., 2008). It is in this context that this novel acute action of individual CSPGs on axonal conduction identified in these experiments should be considered. Our observation that intraspinal injections of NG2, but not neurocan and aggrecan, induced an acute block of axonal conduction suggests that not all CSPGs are harmful to the synaptic projections in adult mammalian spinal cord. This finding carries potential for translational application to strengthen weak projections by manipulating specific CSPGs to enhance conduction of surviving axons in the injured mammalian spinal cord.

\section{References}

Arvanian VL, Mendell LM (2001) Removal of NMDA receptor $\mathrm{Mg}^{2+}$ block extends the action of NT-3 on synaptic transmission in neonatal rat motoneurons. J Neurophys 86:123-129. 
Arvanian VL, Schnell L, Lou L, Golshani R, Hunanyan A, Ghosh A, Pearse DD, Robinson JK, Schwab ME, Fawcett JW, Mendell LM (2009) Chronic spinal hemisection in rats induces a progressive decline in transmission in uninjured fibers to motoneurons. Exp Neurol 216:471-480.

Basso MD, Beattie MS, Bresnahan JC (1995) A sensitive and reliable locomotor rating scale for open field testing in rats. J Neurotrauma 12:1-21.

Bekku Y, Rauch U, Ninomiya Y, Oohashi T (2009) Brevican distinctively assembles extracellular components at the large diameter nodes of Ranvier in the CNS. J Neurochem 108:1266-1276.

Black JA, Waxman SG, Smith KJ (2006) Remyelination of dorsal column axons by endogenous Schwann cells restores the normal pattern of Nav1.6 and Kv1.2 at nodes of Ranvier. Brain 129:1319-1329.

Blight AR (1983) Axonal physiology of chronic spinal cord injury in the cat: intracellular recording in vitro. Neuroscience 4:1471-1486.

Bradbury EJ, Moon LD, Popat RJ, King VR, Bennett GS, Patel PN, Fawcett JW, McMahon SB (2002) Chondroitinase ABC promotes functional recovery after spinal cord injury. Nature 416:636-640.

Busch SA, Silver J (2007) The role of extracellular matrix in CNS regeneration. Curr Opin Neurobiol 17:120-127.

Butt AM, Duncan A, Hornby MF, Kirvell SL, Hunter A, LevineJM, Berry M (1999) Cells expressing the NG2 antigen contact nodes of Ranvier in adult CNS white matter. Glia 26:92-96.

Butt AM, Kiff J, Hubbard P, Berry M (2002) Synantocytes: new functions for novel NG2 expressing glia. J Neurocytol 31:551-565.

Cafferty WB, Bradbury EJ, Lidierth M, Jones M, Duffy PJ, Pezet S, McMahon SB (2008) Chondroitinase ABC-mediated plasticity of spinal sensory function. J Neurosci 28:11998-12009.

Davies JE, Tang X, Denning JW, Archibald SJ, Davies SJ (2004) Decorin suppresses neurocan, brevican, phosphacan and NG2 expression and promotes axon growth across adult rat spinal cord injuries. Eur J Neurosci 19:1226-1242.

Dou CL, Levine JM (1994) Inhibition of neurite growth by the NG2 chondroitin sulfate proteoglycan. J Neurosci 14:7616-7628.

Dours-Zimmermann MT, Maurer K, Rauch U, Stoffel W, Fässler R, Zimmermann DR (2009) Versican V2 assembles the extracellular matrix surrounding the nodes of Ranvier in the CNS. J Neurosci 29:7731-7742.

Galtrey CM, Fawcett JW (2007) The role of chondroitin sulfate proteoglycans in regeneration and plasticity in the central nervous system. Brain Res Rev 54:1-18.

Galtrey CM, Asher RA, Nothias F, Fawcett JW (2007) Promoting plasticity in the spinal cord with chondroitinase improves functional recovery after peripheral nerve repair. Brain 130:926-939.

García-Alías G, Lin R, Akrimi SF, Story D, Bradbury EJ, Fawcett JW (2008) Therapeutic time window for the application of chondroitinase ABC after spinal cord injury. Exp Neurol 210:331-338.

García-Alías G, Barkhuysen S, Buckle M, Fawcett JW (2009) Chondroitinase $\mathrm{ABC}$ treatment opens a window of opportunity for task-specific rehabilitation. Nat Neurosci 12:1145-1151.

Houle JD, Tom VJ, Mayes D, Wagoner G, Phillips N, Silver J (2006) Combining an autologous peripheral nervous system "bridge" and matrix modification by chondroitinase allows robust, functional regeneration beyond a hemisection lesion of the adult rat spinal cord. J Neurosci 26:7405-7415.

Hunanyan AS, García-Alías G, Levine JM, Fawcett JW, Mendell LM, Arvanian VL (2009) Diverse effects of CSPGs on conduction in adult rat spinal cord. Soc Neurosci Abstr 35:365.4.

Iaci JF, Vecchione AM, Zimber MP, Caggiano AO (2007) Chondroitin sulfate proteoglycans in spinal cord contusion injury and the effects of chondroitinase treatment. J Neurotrauma 24:1743-1759.

Iseda T, Okuda T, Kane-Goldsmith N, Mathew M, Ahmed S, Chang YW, Young W, Grumet M (2008) Single, high dose intraspinal injection of chondroitinase reduces glycosaminoglycans in injured spinal cord and promotes corticospinal axonal regrowth after hemisection but not contusion. J Neurotrauma 25:334-349.

Jones LL, Yamaguchi Y, Stallcup WB, Tuszynski MH (2002) NG2 is a major chondroitine sulfate proteoglycan produced after spinal cord injury and is expressed by macrophages and oligodendrocyte progenitors. J Neurosci 22:2792-2803.

Kukley M, Kiladze M, Tognatta R, Hans M, Swandulla D, Schramm J, Dietrich D (2008) Glial cells are born with synapses. FASEB J 22:2957-2969.

Kwok JC, Afshari F, García-Alías G, Fawcett JW (2008) Proteoglycans in the central nervous system: plasticity, regeneration and their stimulation with chondroitinase ABC. Restor Neurol Neurosci 26:131-145.
Laabs TL, Wang H, Katagiri Y, McCann T, Fawcett JW, Geller HM (2007) Inhibiting glycosaminoglycan chain polymerization decreases the inhibitory activity of astrocyte-derived chondroitin sulfate proteoglycans. J Neurosci 27:14494-14501.

Lemons ML, Howland DR, Anderson DK (1999) Chondroitin sulfate proteoglycan immunoreactivity increases following spinal cord injury and transplantation. Exp Neurol 160:51-65.

Lemons ML, Sandy JD, Anderson DK, Howland DR (2001) Intact aggrecan and fragments generated by both aggrecanase and metalloproteinase-like activities are present in the developing and adult rat spinal cord and their relative abundance is altered by injury. J Neurosci 21:4772-4781.

Leonard LJ, Margolis RU, Tuszynski MH (2003) The chondroitin sulfate proteoglycans neurocan, brevican, phosphacan, and versican are differentially regulated following spinal cord injury. Exp Neurol 182:399-411.

Lin R, Kwok JC, Crespo D, Fawcett JW (2008) Chondroitinase ABC has a long lasting effect on chondroitin sulphate glycosaminoglycan content in the injured rat brain. J Neurochem 104:400-408.

Lou L, Mendell LM, Arvanian VL (2008) Enhanced expression pattern of CSPGs rostral and caudal to thoracic lateral hemisection coincides with the reduction in transmission in injured adult rat spinal cord. Soc Neurosci Abstr 34:837.19.

Martin S, Levine AK, Chen ZJ, Ughrin Y, Levine JM (2001) Deposition of the NG2 proteoglycan at nodes of Ranvier in the peripheral nervous system. J Neurosci 21:8119-8128.

Massey JM, Amps J, Viapiano MS, Matthews RT, Wagoner MR, Whitaker CM, Alilain W, Yonkof AL, Khalyfa A, Cooper NG, Silver J, Onifer SM (2008) Increased chondroitin sulfate proteoglycan expression in denervated brainstem targets following spinal cord injury creates a barrier to axonal regeneration overcome by chondroitinase ABC and neurotrophin-3. Exp Neurol 209:426-445.

Melendez-Vasquez C, Carey DJ, Zanazzi G, Reizes O, Maurel P, Salzer JL (2005) Differential expression of proteoglycans at central and peripheral nodes of Ranvier. Glia 52:301-308.

Paukert M, Bergles DE (2006) Synaptic communication between neurons and NG2+ cells. Curr Opin Neurobiol 16:515-521.

Pizzorusso T, Medini P, Berardi N, Chierzi S, Fawcett JW, Maffei L (2002) Reactivation of ocular dominance plasticity in the adult visual cortex. Science 298:1248-1251.

Reed WR, Shum-Siu A, Magnuson DS (2008) Reticulospinal pathways in the ventrolateral funiculus with terminations in the cervical and lumbar enlargements of the adult rat spinal cord. Neuroscience 151:505-517.

Rolls A, Shechter R, Schwartz M (2009) The bright side of the glial scar in CNS repair. Nat Rev Neurosci 10:235-241.

Schwartz NB, Domowicz M, Krueger RC Jr, Li H, Mongoura D (1996) Brain aggrecan. Perspect Dev Neurobiol 3:291-306.

Snow DM, Lemmon V, Carrino DA, Caplan AI, Silver J (1990) Sulfated proteoglycans in astroglial barriers inhibit neurite outgrowth in vitro. Exp Neurol 109:111-130.

Tan AM, Colletti M, Rorai AT, Skene JH, Levine JM (2006) Antibodies against the NG2 proteoglycan promote the regeneration of sensory axons within the dorsal columns of the spinal cord. J Neurosci 26:4729-4739.

Tang X, Davies JE, Davies SJ (2003) Changes in distribution, cell associations, and protein expression levels of NG2, neurocan, phosphacan, brevican, versican V2, and tenascin-C during acute to chronic maturation of spinal cord scar tissue. J Neurosci Res 71:427-444.

Teng X, Nagata I, Li HP, Kimura-Kuroda J, Sango K, Kawamura K, Raisman G, Kawano H (2008) Regeneration of nigrostratial dopaminergic axons after transplantation of olfactory ensheathing cells and fibroblast prevents fibrotic scar formation at the lesion site. J Neurosci Res 86:3140-3150.

Uhgrin Y, Chen ZJ, Levine JM (2003) Multiple domains of the NG2 proteoglycan mediate axon growth inhibition. J Neurosci 23:175-186.

Wang X, Messing A, David S (1997) Axonal and nonneuronal cell responses to spinal cord injury in mice lacking glial fibrillary acidic protein. Exp Neurol 148:568-576.

Wigley R, Hamilton N, Nishiyama A, Krichhoff F, Butt AM (2007) Morphological and physiological interactions of NG2-glia with astrocytes and neurons. J Anat 210:661-670.

Yamaguchi Y (2000) Lecticans: organizers of the brain extracellular matrix. Cell Mol Life Sci 57:276-289. 